\section{Christian S. Kessler ${ }^{\mathrm{a}}$ \\ Elmar Stapelfeldt ${ }^{a}$ \\ Andreas Michalsen $^{\mathrm{a}}$}

Angenommen, Sie gehören zu den Ärzten oder Ärztinnen, die sich beruflich schon länger etwas Abwechslung wünschen. Möglicherweise liebäugeln Sie seit Jahren damit, für «Ärzte ohne Grenzen» in einem subtropischen Land $\mathrm{zu}$ arbeiten. Oder Sie hatten schon immer ein Faible für Exotisches in Ihrem Leben. Vielleicht haben Sie dann Lust, einen Einblick in Ayurveda zu erhalten, die Traditionelle Indische Medizin. Falls dem so sein sollte, laden wir Sie herzlich ein, mit uns virtuell in einem Airbus nach Indien zu starten, Neu Delhi als Ziel, ca. 8 Flugstunden von der Schweiz entfernt, um sich auf eine kleine Reise zu begeben.

Sie sind unterwegs zu einem zweiwöchigen Crashkurs für Tropenmedizin der World Health Organization (WHO), den Sie schon seit dem Studium machen wollten. Ihre Arbeit in der Praxis war in letzter Zeit extrem anstrengend. Ausserdem können Sie ein wenig Abstand vertragen, da die fortschreitende rheumatische Polyarthritis ihres Vaters immer mehr Probleme in der Familie aufwirft. Während Sie in der Economy Class das vegetarische Menü (wie ungewöhnlich für eine grosse Airline!) verspeisen, blättern Sie im In-FlightMagazin und bleiben an einem verschwenderischen Artikel über Ayurveda-Wellness in Südindien hängen, weil Ihnen eine Freundin mit der Zusatzbezeichnung Naturheilkunde erst vor kurzem mit ihrer Ayurveda-

\footnotetext{
${ }^{a}$ Abteilung für Naturheilkunde, Immanuel Krankenhaus Berlin-Wannsee, Berlin, Deutschland.
}

\title{
Für Ärzte mit Vorstellungsvermögen - Traditionelle Indische Medizin: Ayurveda
}

Kur in Kerala in den Ohren gelegen hatte. Sie erinnern sich gut daran, dass Ihnen die Schwärmerei von Ayurveda als ganzheitliche indische Naturheilkunde mit all den Ölmassagen, Stirngüssen, Kräutereinläufen und Yoga-Übungen reichlich kitschig und shanti vorgekommen war, zumal die Kollegin nur einen Entspannungsurlaub gebucht hatte. Sie selber hatten bislang nur von Schwermetallbelastungen in Ayurveda-Präparaten gelesen [1]. Urlaubswahn wahrscheinlich!

Touchdown in Delhi. Als Mitbringsel aus der Schweiz haben Sie eine saftige Erkältung eingeschleppt, die im tropischen Klima so richtig durchkommt. Der freundliche Rikscha-Fahrer Raj hat Ihr Niesen sofort registriert und gibt Ihnen als selbstbekundeter Neffe eines AyurvedaArztes den Rat, bis zum Abklingen Ihrer Beschwerden dreimal täglich einen Teelöffel Kurkuma-Pulver in etwas lauwarmem Wasser gelöst einzunehmen [2]. Zusammen mit reichlich heissem Ingwerwasser sei das bei Erkältungen ein echtes Wundermittel und erspare den Gang zur Apotheke [3]. Garantiert. Na dann!

Ihre zwei Eingewöhnungstage - Kulturschock Indien! - verbringen Sie am Dachterrassenpool Ihres Hotels über dem Dunst von Delhi. Bemerkenswerterweise hat das Restaurant neben den üblichen Verdächtigen wie Chicken Madras und Alu Gobi auch Ayurveda-Gerichte entsprechend den Prinzipien von Vata, Pitta und Kapha [4-6] im Angebot - was auch immer das heissen mag! Als Sie Lamm Vindaloo bestellen wollen, schaut Sie der Koch, Ihren Taschentuchhaufen vor sich im Blick, etwas mitleidig an und empfiehlt Ihnen, bei Erkältung doch eher auf die leichtverdauliche Reis-Mung-LinsenVariante auszuweichen; das würde kräftigen, ohne zu belasten. Die britische Langzeittouristin am Tisch nebenan pflichtet ihm bei und kramt für Sie zwei Päckchen Sitopaladi Ayurveda Anti-Cold, die sie in der Ayurveda-Pharmacy in den Chowks erstanden habe, aus ihrem Täschchen. Eigentlich lecker: süss und scharf!

Der Tropenkurs ist klasse! Eine überfällige Abwechslung zum täglichen Trott in der Praxis. Auf der Rückfahrt vom Regionalbüro der WHO bemerken Sie ein grosses Gebäude, auf dem «Central Council for Research in Ayurvedic Sciences (CCRAS)» steht [7]. Ihr Fahrer erklärt Ihnen, dass das Gelände zum indischen Gesundheitsministerium gehöre und sich dort die Forschungsund Verwaltungseinrichtungen für die Traditionelle Indische Medizin befänden. Als Sie Ihren Kursleiter Prof. Sukhadev am nächsten Tag darauf ansprechen, erklärt er Ihnen, dass Ayurveda in Südasien eine Breitenmedizin sei und in Indien neben fünf weiteren Medizinsystemen der Schulmedizin gesetzlich gleichgestellt wäre - mit über 400000 approbierten Ärzten [8] und mehr als 250 akademischen Lehrinstituten [9]. Als indischer Mitarbeiter betont er stolz, dass Ayurveda von der WHO vor längerer Zeit anerkannt wurde [10]. Wissen tue er das auch erst, seitdem er 2006 in einer internationalen tropenmedizinischen Arbeitsgruppe der WHO zur Eindämmung des Chikungunya-Fiebers mit einem Ayurveda-Professor an einem

\section{KARGER}

Fax +497614520714 Information@Karger.com www.karger.com
(๑) 2013 S. Karger GmbH, Freiburg

Accessible online at: www.karger.com/szg
Dr. med. Christian S. Kessler, M.A.

Forschungskoordination, Abteilung für Naturheilkunde

Immanuel Krankenhaus Berlin-Wannsee

Königstrasse 63, 14109 Berlin, Deutschland

c.kessler@immanuel.de 
Tisch gesessen habe. Interessant, wie die Systeme hier ineinanderzugreifen scheinen!

Da sie aufgeschlossen gegenüber Ayurveda wirken, macht er Sie mit einem österreichischen Teilnehmer aus dem Kurs bekannt, Dr. Pabst, Allgemeinmediziner aus Salzburg, der sich in seiner Praxis seit längerem mit Ayurveda beschäftigt. Der Kollege berichtet, wie er Ayurveda-Therapieelemente fast täglich in seine integrativmedizinischen Konzepte einfliessen lasse, und klagt dabei über die zum Teil noch unzureichende Erstattungsfähigkeit im deutschsprachigen Raum. Vielleicht könne der Verband Europäischer Ayurveda Therapeuten (VEAT) [11], bei dem er gerade Mitglied geworden sei, noch etwas dazu beitragen, vor allem auch im ärztlichen Bereich. Das wäre ein weiterer Fortschritt, denn bei seinen Patienten kämen gerade die Selbstwirksamkeitsaspekte von Ayurveda sehr gut an [12]. Sie denken an Ihren Vater. «Zur rheumatoiden Arthritis und Ayurveda gibt es eine hochinteressante Studie aus den USA. Klicken Sie doch mal rein!», sagt Dr. Pabst [13].

Eigentlich wollten Sie an Ihrem ersten Wochenende in Delhi die Sehenswürdigkeiten abhaken, aber der Salzburger Kollege hat am Wochenende einen Ausflug $\mathrm{zu}$ einem renommierten Ayurveda College in Jaipur, Rajasthan, organisiert, etwa 4 Autostunden von Delhi entfernt. Dort möchte er einen seiner Lehrer besuchen und hat Sie spontan eingeladen, ihn zu begleiten. Zu Ihrer eigenen Überraschung sagen Sie zu. In dem Ayurveda-Lehrkrankenhaus mit 120 Betten erwartet Sie kein überbordender Ayurveda-Luxus. Die Ausstattung ist eher Holzklasse und die Patienten, einige von ihnen auch Europäer, kommen nicht zum Wellness-Urlaub, sondern handfester gesundheitlicher Probleme wegen. Der Chefarzt der internistischen Ayur-
veda-Abteilung, Prof. Singh, reist mehrmals im Jahr als Dozent im Rahmen von Master-Studiengängen zu europäischen Ayurveda-Akademien nach Grossbritannien, Deutschland und in die Schweiz [14]. Prof. Singh fragt Sie, ob Sie schon von der klinischen Ayurveda-Studie zu Kniegelenksarthrose gehört hätten, die gerade an der Charité Naturheilkunde in Berlin durchgeführt und vom indischen Gesundheitsministerium finanziert werde [15]. Es wäre doch erstaunlich, was in der globalisierten Welt heute alles möglich sei!

Beim Mittagessen in der Kantine treffen Sie völlig unerwartet auf eine junge Medizinstudentin aus Hannover, die in der schulmedizinischen Klinik nebenan einen Teil ihres PJTertials Chirurgie absolviert und in ihrer Freizeit bei Prof. Singh hospitiert. Seit 1,5 Jahren arbeitet sie an ihrer Doktorarbeit - eine systematische Übersichtsarbeit mit Metaanalyse zu Ayurveda bei rheumatischen Erkrankungen. Hierfür würde sie neben Pubmed vor allem auch die digitale wissenschaftliche AyurvedaDatenbank Dhara verwenden, die kürzlich online gegangen ist und mittlerweile über 55000 Einträge aufweist [16]. Zum Abschluss lädt sie Sie und Dr. Pabst ein, im November zu einem Ayurveda-Symposium an die indische Botschaft in Bern zu kommen, um sich selbst einen Überblick über die Ayurveda-Aktivitäten in der Schweiz und in Europa zu verschaffen.

Drei Wochen später kommt Ihr Aufenthalt in Indien mit einer Rundreise durch Nordindien $\mathrm{zu}$ seinem Ende. Sie sitzen wieder in der Economy Class von Air India nach Zürich und reflektieren den Tropenkurs sowie Ihre ziemlich unerwarteten Ayurveda-Begegnungen. Ihre Gedanken kreisen um Ihren Praxisalltag, der Sie übermorgen wieder einholen wird - möglicherweise ab demnächst mit Ayurveda-Medizin?

\section{Literatur}

1 Desai A, Staszewski H: Ayurvedic remedy for diabetes as a cause of lead poisoning: a case report. Am J Med 2012;125:e3-4.

2 Menon V, Sudheer A: Antioxidant and antiinflammatory properties of curcumin. Adv Exp Med Biol 2007;595:105.

3 Kim JK, Kim Y, Na KM, Surh YJ, Kim TY: [6]-Gingerol prevents UVB-induced ROS production and COX-2 expression in vitro and in vivo. Free Radic Res 2007;41:603614.

4 Gupta SN, Stapelfeldt E: Praxis der Ayurveda-Medizin. Kaya-cikitsa. Therapiekonzepte für innere Erkrankungen. Stuttgart, Haug, 2009, pp 10-12, pp 201-209.

5 Lad V: Lehrbuch des Ayurveda. Die Grundprinzipien, Band 1, ed 1. Kandern, Narayana, 2012.

-6 Schrott E, Ammon HPT: Heilpflanzen der ayurvedischen und der westlichen Medizin. Heidelberg, Springer, 2012.

7 Central Council for Research in Ayurvedic Science, Department of AYUSH, Ministry of Health and Family Welfare, Government of India. www.ccras.nic.in.

8 Association of Ayurvedic Physicians of India (AAPI). http://aapiindia.org.

9 Kessler C: Wirksamkeit von Ayurveda bei chronischen Erkrankungen. Systematische Analysen klinischer Ayurveda-Studien. Essen, KVC, 2007.

10 Bodeker G, Ong CK, Grundy C, Burford E, Shein K: WHO, Global Atlas of Traditional, Complementary and Alternative Medicine. Kobe, World Health Organisation, 2005. WHO Traditional Medicine Strategy 20022005. Geneva, WHO, 2002. www.who.int/ gb/ebwha/pdf_files/WHA56/ea5618.pdf.

11 Verband Europäischer Ayurveda Therapeuten (VEAT). www.ayurveda-verband.eu.

12 Kessler C, Michalsen A: The role of whole medical systems in global medicine. Forsch Komplementmed 2012;19:65-66.

13 Furst DE, Venkatraman MM, McGann M, Manohar PR: Double-blind, randomized, controlled pilot study comparing classic ayurvedic medicine, methotrexate and their combination in rheumatoid arthritis. J Clin Rheumatol 2011;17:185-192.

14 Europäische Akademie für Ayurveda. www.ayurveda-akademie.org.

15 Michalsen A, Kessler C, Witt C: Complex Ayurvedic Treatment for Osteoarthritis of the Knee (CARAKA-Trial) - Design of a Randomized Controlled Trial Comparing Ayurveda and Conventional Standard Care Including a Diagnostic Pilot Study (PARIKSA-Study). Abstract Book of the International Congress on Complementary Medicine Research in Chengdu, China. May 2011

16 Digital Helpline for Ayurveda Research Articles (DHARA). www.dharaonline.org. 